\title{
A Theoretical and Experimental Investigation into Pair-induced Quenching in Bismuth Oxide-based Erbium-doped Fiber Amplifiers
}

\author{
Minwan Jung ${ }^{1}$, Jae Hyun Shin ${ }^{1}$, Young Min Jhon ${ }^{2}$, and Ju Han Lee ${ }^{1 *}$ \\ ${ }^{I}$ School of Electrical and Computer Engineering, University of Seoul, \\ 13 Siripdae-gil, Dongdaemun-gu, Seoul 130-743, Korea \\ ${ }^{2}$ Photonics/Sensor System Center, Korea Institute of Science and Technology, Seoul 136-791, Korea
}

(Received October 1, 2010 : revised November 1, 2010 : accepted November 23, 2010)

\begin{abstract}
The pair-induced quenching (PIQ) effect in a highly doped bismuth oxide-based erbium-doped fiber amplifier (EDFA) was theoretically and experimentally investigated. In the theoretical investigation, the bismuth oxide-based EDFA was modeled as a 6-level amplifier system that incorporated clustering-induced concentration quenching, cooperative up-conversion, pump excited state absorption (ESA), and signal ESA. The relative number of paired ions in a highly doped bismuth oxide EDF was estimated to be $\sim 6.02 \%$, determined by a comparison between the theoretical and the experimentally measured gain values. The impacts of the PIQ on the gain and the noise figure were also investigated.
\end{abstract}

Keywords : Erbium-doped Fibers, Bismuth Oxide, Quenching, Clustering

OCIS codes : (060.2410) Fibers, Erbium; (060.2320) Fiber optics amplifiers and oscillators; (060.2330)

Fiber optics communications

\section{INTRODUCTION}

Erbium-doped fiber amplifiers are essential devices in current high capacity fiber-optic telecommunication systems that are based on wavelength division multiplexing (WDM) technology [1]. Commonly used silica-based EDFAs are known to provide sufficient signal gain over an amplification bandwidth of $\sim 30 \mathrm{~nm}$ in the $\mathrm{C}$ or the $\mathrm{L}$ bands, depending on the configuration. Even though the conventional silica-based EDFAs are well-suited for current WDM systems, there has been extensive work done in order to increase the EDFA gain bandwidth, due to the ever increasing demand for the expansion of data transmission capacity that has been caused by the large increase in the amount of data traffic on the Internet.

Ultra-wideband EDFAs that can cover a gain bandwidth of more than $50 \mathrm{~nm}$ have been successfully demonstrated using EDFs based on non-silica host materials, such as tellurite [2], bismuth oxide [3], and antimony silicate [4]. Among the non-silica host material-based EDFs, the tellurite and bismuth oxide based ones are known to be capable of providing sufficient signal gain over the $\mathrm{C}$ and the $\mathrm{L}$ bands. The demonstrated gain bandwidth of tellurite and bismuth oxide EDFAs was $\sim 70 \mathrm{~nm}$, whereas the bandwidth of the antimony silicate EDFAs was $\sim 50 \mathrm{~nm}$. Another benefit of the tellurite and bismuth oxide EDFs is found in their high erbium doping concentration, which is enabled by the host materials' high solubility to erbium ions. This feature is useful in the creation of compact fiber amplifiers with short fiber lengths.

Compared to both the antimony silicate-based and the tellurite-based EDFs, a huge benefit in using bismuth oxidebased EDFs is the fact that they can be readily spliced to conventional silica fibers using a commercially available fusion splicer with a splicing loss of less than $0.5 \mathrm{~dB}$ [3]. This shows that bismuth oxide EDFs are superior to the other new host materials in terms of the ease of integration with pre-existing silica fiber-based communication systems.

One concern in realizing high-performance ultra-broadband amplifiers using bismuth oxide EDFs is the limitation in the amplification performance due to the various inhomogeneous and nonlinear effects caused by the high erbium concentration and the large host material nonlinearity. A range of experimental and theoretical studies on such effects as four wave mixing [3], cooperative up-conversion [5], pump excited state absorption (ESA) [6], and signal ESA [7] have been

*Corresponding author: j.h.lee@ieee.org 
carried out.

In addition to the above-mentioned effects, another important effect that needs to be investigated is the pairinduced quenching (PIQ). The PIQ is an inhomogeneous phenomenon caused by clustered ions when the $\mathrm{Er}^{3+}$ ions are not evenly distributed. The PIQ is known to be strongly dependent on the host material; it is always observed in highly $\mathrm{Er}^{3+}$-doped fibers [8]. In an ion pair, one ion (donor) transfers its energy to the other (acceptor) when both of them are excited. The donor ion relaxes to its ground state, whereas the acceptor ion jumps to a higher energy level and relaxes back to its metastable state [9]. The PIQ can thereby cause EDFA performance degradation, such as gain reduction.

In this paper, we investigate the PIQ effect in a bismuth oxide-based EDFA in both a theoretical and an experimental manner. In the theoretical analysis we used a 6-level amplifier model that incorporates cooperative upconversion, PIQ, pump ESA, and signal ESA. In a comparison between the numerically calculated and experimentally measured gain and noise figure (NF) values, the relative number of paired ions is estimated to be $\sim 6.02 \%$ in a commercially available bismuth oxide-based EDF. The impacts of the PIQ on the gain and the NF were also investigated.

This paper is organized as follows: In Section II the theoretical model used for this investigation is presented. In Section III the relative number of ion pairs in a commercially available bismuth oxide EDF is estimated, by comparing the numerically calculated gain and NF values with the experimentally measured ones. In Section IV, the PIQ is shown to be a significant degradation factor in the gain and NF performance. This is done by comparing the numerical and experimental output gains both with and without the PIQ. In Section V our conclusions are drawn from the obtained results.

\section{THEORETICAL MODELING OF A BISMUTH OXIDE-BASED EDFA}

For the numerical simulation, the bismuth oxide EDFA was assumed to be a six level system composed of the ${ }^{4} I_{15 / 2},{ }^{4} I_{13 / 2},{ }^{4} I_{11 / 2},{ }^{4} I_{9 / 2},{ }^{4} S_{3 / 2}$, and ${ }^{4} F_{7 / 2}$ states, as shown in Fig. 1. The EDFA model included the PIQ effects, the homogeneous cooperative up-conversion, the pump ESA, and the signal ESA [7], [10-13]. In the model, the effects of the pump $\left(P_{p}\right)$, signal $\left(P_{s}\right)$, and amplified spontaneous emission (ASE) $\left(P_{A S E}\right)$ power on the populations in the energy level system were represented by the following two separate rate equations. The clustered ions were assumed to be pairs.

The Singled Ion Rate Equations

$$
\frac{d N_{1}^{S}}{d t}=-\left(R_{13}+R_{12}+W_{12}\right) N_{1}^{S}+\left(A_{21}+W_{21}+R_{21}+C_{u p} N_{2}^{S}\right) N_{2}^{S}+R_{31} N_{3}^{S}+A_{51} N_{5}^{S}
$$

$\frac{d N_{2}^{S}}{d t}=\left(R_{12}+W_{12}\right) N_{1}^{S}-\left(A_{21}+W_{21}+R_{21}+2 C_{u p} N_{2}^{S}+W_{24}^{E S A}\right) N_{2}^{S}+A_{32} N_{3}^{S}$

$\frac{d N_{3}^{S}}{d t}=R_{13} N_{1}^{S}-\left(A_{32}+R_{31}+R_{36}^{E S A}\right) N_{3}^{S}+A_{43} N_{4}^{S}$

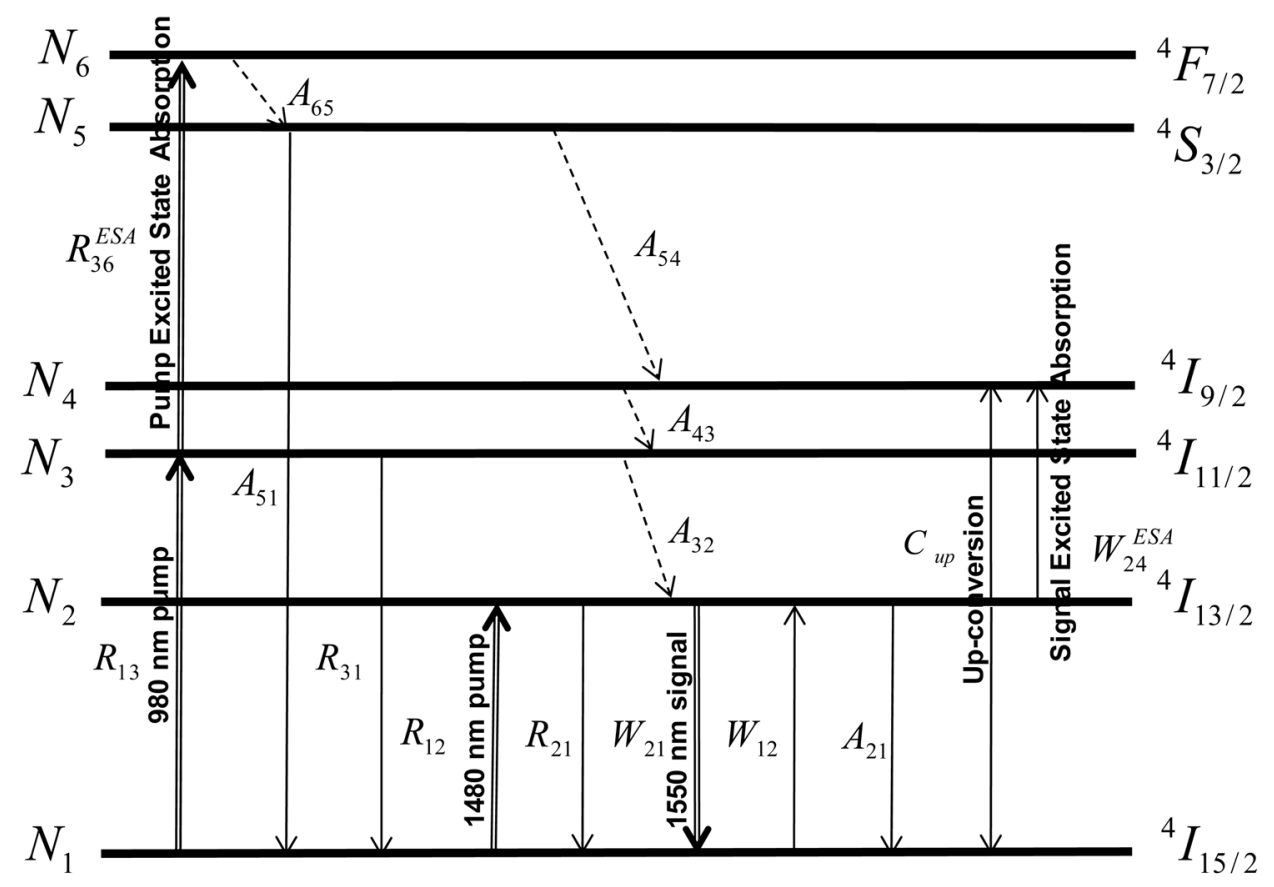

FIG. 1. The schematic diagram of the energy levels and the erbium transition in bismuth oxide glass. 


$$
\begin{aligned}
& \frac{d N_{4}^{S}}{d t}=\left(C_{u p} N_{2}^{S}+W_{24}^{E S A}\right) N_{2}^{S}-A_{43} N_{4}+A_{54} N_{5}^{S} \\
& \frac{d N_{5}^{S}}{d t}=-\left(A_{51}+A_{54}\right) N_{5}^{S}+A_{65} N_{6}^{S} \\
& \frac{d N_{6}^{S}}{d t}=R_{36}^{E S A} N_{3}^{S}-A_{65} N_{6}^{S} \\
& N_{1}^{S}+N_{2}^{S}+N_{3}^{S}+N_{4}^{S}+N_{5}^{S}+N_{6}^{S}=(1-m k) N_{t}
\end{aligned}
$$

where $N_{1}^{s}, N_{2}^{s}, N_{3}^{s}, N_{4}^{s}, N_{5}^{s}$, and N6s represent the number of singled erbium ions in the ${ }^{4} I_{15 / 2},{ }^{4} I_{13 / 2},{ }^{4} I_{11 / 2},{ }^{4} I_{9 / 2},{ }^{4} S_{3 / 2}$, and ${ }^{4} F_{7 / 2}$ states, respectively. $m$ is the number of ions within a cluster ( 2 for paired ions) and $k$ is the relative number of clusters. $A_{21}$ and $A_{51}$ are the spontaneous emission rates from ${ }^{4} I_{13 / 2}$ and ${ }^{4} S_{3 / 2}$ to ${ }^{4} I_{15 / 2}$, respectively. $C_{u p}$ is the up-conversion coefficient. $A_{32}$ is the non-radiative decay rate from the ${ }^{4} I_{11 / 2}$ state to ${ }^{4} I_{13 / 2}$, whereas $A_{43}$ is the non-radiative decay rate from ${ }^{4} I_{9 / 2}$ to ${ }^{4} I_{11 / 2} . A_{54}$ is the non-radiative decay rate from the ${ }^{4} S_{3 / 2}$ state to ${ }^{4} I_{9 / 2}$, whereas $A_{65}$ is the non-radiative decay rate from ${ }^{4} F_{7 / 2}$ to ${ }^{4} S_{3 / 2} . R_{13}$ and $R_{31}$ are the pump absorption and stimulated emission rates for $980 \mathrm{~nm}$ pumping, and $R_{12}$ and $R_{21}$ are the pump absorption and stimulated emission rates for 1480-nm pumping. $W_{12}, W_{21}$ are the signal absorption and stimulated emission rates. $R_{36}{ }^{E S A}$ and $W_{24}{ }^{E S A}$ are the pump and signal ESA rates, respectively. These rates are given in the following forms:

$$
\begin{aligned}
& R_{12}=\left(\frac{P_{p}^{+}+P_{p}^{-}}{h v_{p} \pi r_{m}{ }^{2}}\right) \Gamma_{p} \sigma_{p a 2} \\
& R_{13}=\left(\frac{P_{p}^{+}+P_{p}^{-}}{h v_{p} \pi r_{m}{ }^{2}}\right) \Gamma_{p} \sigma_{p a} \\
& R_{21}=\left(\frac{P_{p}^{+}+P_{p}^{-}}{h v_{p} \pi r_{m}{ }^{2}}\right) \Gamma_{p} \sigma_{p e 2} \\
& R_{31}=\left(\frac{P_{p}^{+}+P_{p}^{-}}{h v_{p} \pi r_{m}{ }^{2}}\right) \Gamma_{p} \sigma_{p e} \\
& W_{12}=\left(\frac{P_{S}+P_{A S E}{ }^{+}+P_{A S E}{ }^{-}}{h v_{s} \pi r_{m}{ }^{2}}\right) \Gamma_{s} \sigma_{s a} \\
& W_{21}=\left(\frac{P_{S}+P_{A S E}{ }^{+}+P_{A S E}{ }^{-}}{h v_{s} \pi r_{m}{ }^{2}}\right) \Gamma_{s} \sigma_{s e} \\
& R_{36}^{E S A}=\left(\frac{P_{p}^{+}+P_{p}^{-}}{h v_{p} \pi r_{m}{ }^{2}}\right) \Gamma_{p} \sigma_{P u m p}^{E S A} \\
& W_{24}^{E S A}=\left(\frac{P_{A S E}{ }^{+}+P_{A S E}{ }^{-}}{h v_{s} \pi r_{m}{ }^{2}}\right) \Gamma_{s} \sigma_{S i g n a l}^{E S A}
\end{aligned}
$$

The superscripts of + and - indicate the relative beam propagation direction. $\sigma_{p a}$ and $\sigma_{p e}$ represent the absorption (a) and stimulated emission (e) cross-sections of the 980-nm pump, and $\sigma_{p a 2}$ and $\sigma_{p e z}$ indicate the absorption $(a)$ and stimulated emission (e) cross-sections of the 1480-nm pump, respectively. $\sigma_{s a}$ and $\sigma_{s e}$ are the absorption $(a)$ and stimulated emission (e) cross-sections of the signal $(s) . \sigma_{\text {Signal }}^{E S A}$ is the signal ESA cross-section. $\Gamma_{s}$ and $\Gamma_{p}$ are the signal-to-core and pump-to-core overlap factor, respectively. $r_{m}$ is the mode field radius of the EDF. $h$ is Planck's constant, $v_{p}$ is the pump frequency and $v_{s}$ is the signal frequency.

\section{The Paired Ion Rate Equations}

$$
\begin{aligned}
& \frac{d N_{1}^{P}}{d t}=-\left(R_{13}+R_{12}+W_{12}\right) N_{1}^{P}+\left(A_{21}+W_{21}+W_{12}+R_{13}+R_{21}+R_{12}\right) N_{2}^{P}+R_{31} N_{3}^{P}+A_{51} N_{5}^{P} \\
& \frac{d N_{2}^{P}}{d t}=\left(R_{12}+W_{12}\right) N_{1}^{P}-\left(A_{21}+W_{21}+W_{12}+R_{21}+R_{12}+R_{13}+W_{24}^{E S A}\right) N_{2}^{P}+A_{32} N_{3}^{P} \\
& \frac{d N_{3}^{P}}{d t}=R_{13} N_{1}^{P}-\left(A_{32}+R_{31}+R_{36}^{E S A}\right) N_{3}^{P}+A_{43} N_{4}^{P} \\
& \frac{d N_{4}^{P}}{d t}=W_{24}^{E S A} N_{2}^{P}-A_{43} N_{4}^{P}+A_{54} N_{5}^{P} \\
& \frac{d N_{5}^{P}}{d t}=-\left(A_{51}+A_{54}\right) N_{5}^{P}+A_{65} N_{6}^{P} \\
& \frac{d N_{6}^{P}}{d t}=R_{36}^{E S A} N_{3}^{P}-A_{65} N_{6}^{P} \\
& N_{1}^{P}+N_{2}^{P}+N_{3}^{P}+N_{4}^{P}+N_{5}^{P}+N_{6}^{P}=m k N_{t}
\end{aligned}
$$

where $N_{1}^{p}, N_{2}^{p}, N_{3}^{p}, N_{4}^{p}, N_{5}^{p}$, and $N_{6}^{p}$ represent the number of paired erbium ions in the ${ }^{4} I_{15 / 2},{ }^{4} I_{13 / 2},{ }^{4} I_{11 / 2},{ }^{4} I_{9 / 2},{ }^{4} S_{3 / 2}$, and ${ }^{4} F_{7 / 2}$ states, respectively. The absorption, stimulated emission, and spontaneous emission rates were assumed to be the same for the singled and paired ions. The signal ESA rate was also assumed to be the same for both cases. Subsequently, the total doping concentration $N_{t}$ is given by:

$$
\begin{aligned}
& N_{1}^{S}+N_{1}^{P}=N_{1} \\
& N_{2}^{S}+N_{2}^{P}=N_{2} \\
& N_{3}^{S}+N_{3}^{P}=N_{3} \\
& N_{4}^{S}+N_{4}^{P}=N_{4} \\
& N_{5}^{S}+N_{5}^{P}=N_{5} \\
& N_{6}^{S}+N_{6}^{P}=N_{6} \\
& N_{1}+N_{2}+N_{3}+N_{4}+N_{5}+N_{6}=N_{t}
\end{aligned}
$$

where $N_{l}, N_{2}, N_{3}, N_{4}, N_{5}$, and $N_{6}$ are the number of total erbium ions in the ${ }^{4} I_{15 / 2},{ }^{4} I_{13 / 2},{ }^{4} I_{11 / 2},{ }^{4} I_{9 / 2},{ }^{4} S_{3 / 2}$, and ${ }^{4} F_{7 / 2}$ states, respectively.

The evolution of the pump, signal, and ASE powers along the EDF length can be described through the following propagation equations:

\section{The Propagation Equations}

$$
\frac{d P_{p}^{ \pm}}{d z}=\mp\left(\sigma_{p a 2} N_{1}+\sigma_{p a} N_{1}-\sigma_{p e 2} N_{2}-\sigma_{p e} N_{3}+\sigma_{P \text { sump }}^{E S A} N_{3}\right) P_{p}^{ \pm} \mp \alpha_{p} P_{p}
$$




$$
\begin{aligned}
& \frac{d P_{s}}{d z}=\left(\sigma_{s e} N_{2}-\sigma_{s a} N_{1}-\sigma_{\text {Signal }}^{E S A} N_{2}\right) P_{s}-\alpha_{s} P_{s} \\
& \frac{d P_{A S E}^{ \pm}}{d z}= \pm\left(\sigma_{s e} N_{2}-\sigma_{s a} N_{1}-\sigma_{\text {Signal }}^{E S A} N_{2}\right) P_{A S E}^{ \pm} \pm 2 \sigma_{s e} N_{2} h v_{s} \Delta v \mp \alpha_{s} P_{A S E}^{ \pm}
\end{aligned}
$$

where $\Delta v$ is the spectral width of the ASE at $v_{s} . \alpha_{p}$ and $\alpha_{s}$ are the pump and signal propagation losses, respectively. The propagation equations were numerically solved with the population information at each level, which was obtained under steady state conditions.

The initial conditions for the numerical calculation are as follows:

\section{The Initial Conditions}

$$
\begin{aligned}
& P_{p+}(z=0)=P_{p+}, \quad P_{p-}(z=L)=P_{p-} \\
& P_{s}\left(z=0, v_{s}\right)=P_{s}\left(v_{s}\right) \\
& P_{A S E+}(z=0, v)=P_{A S E-}(z=L, v)=0
\end{aligned}
$$

where $L$ is the length of the EDF used.

The signal absorption and emission cross-sections used in this work were obtained from $[14,15]$. The pump ESA Cross-section was assumed to be $\sigma_{\text {pump }}^{E S A} \approx 2 \times \sigma_{p e}$ as seen in [6]. Fig. 2 shows the stimulated emission, absorption, and signal ESA cross-sections used in this investigation. The

\begin{tabular}{|c|c|c|c|}
\hline Parameter & Symbol & Unit & Value \\
\hline \multirow{2}{*}{ Spontaneous Emission Rate } & $A_{21}^{\mathrm{a}}$ & $1 / \mathrm{s}$ & 300 \\
\hline & $A_{51}{ }^{\mathrm{b}}$ & $1 / \mathrm{s}$ & 3,100 \\
\hline \multirow{4}{*}{ Nonradiative Decay Rate } & $A_{32}{ }^{\mathrm{a}}$ & $1 / \mathrm{s}$ & 7,500 \\
\hline & $A_{43}{ }^{\mathrm{c}}$ & $1 / \mathrm{s}$ & $\sim 1 \times 10^{9}$ \\
\hline & $A_{54}^{\mathrm{b}}$ & $1 / \mathrm{s}$ & 370,000 \\
\hline & $A_{65}{ }^{\mathrm{d}}$ & $1 / \mathrm{s}$ & $\sim 39237$ \\
\hline Upconversion Rate & $C_{u p}{ }^{\mathrm{e}}$ & $\mathrm{m}^{3} / \mathrm{s}$ & $2.2 \times 10^{-24}$ \\
\hline Peak Emission Cross-section & $\sigma_{s a}{ }^{\mathrm{f}}$ & $\mathrm{m}^{2}$ & $9.43 \times 10^{-25}$ \\
\hline Peak Absorption Cross-section & $\sigma_{s a}^{\mathrm{f}}$ & $\mathrm{m}^{2}$ & $8.31 \times 10^{-25}$ \\
\hline Pump Overlap Factor for $1480 \mathrm{~nm}$ & $\Gamma_{p}^{\mathrm{a}}$ & & 0.5 \\
\hline Pump Overlap Factor for $980 \mathrm{~nm}$ & $\Gamma_{p}^{\mathrm{b}}$ & & 0.52 \\
\hline Signal Overlap Factor & $\Gamma_{s}^{\mathrm{a}}$ & & 0.47 \\
\hline Signal Propagation Loss & $a_{s}^{\mathrm{g}}$ & $\mathrm{dB} / \mathrm{m}$ & 0.7 \\
\hline Pump Propagation Loss & $a_{p}^{\mathrm{g}}$ & $\mathrm{dB} / \mathrm{m}$ & 0.7 \\
\hline Density of Erbium Ions & $N_{t}^{\mathrm{a}}$ & $\mathrm{m}^{-3}$ & $7.9 \times 10^{25}$ \\
\hline Mode Field Radius & $r_{m}^{\mathrm{g}}$ & $\mu \mathrm{m}$ & 6.2 \\
\hline Fiber Length & & $\mathrm{m}$ & 2 \\
\hline
\end{tabular}

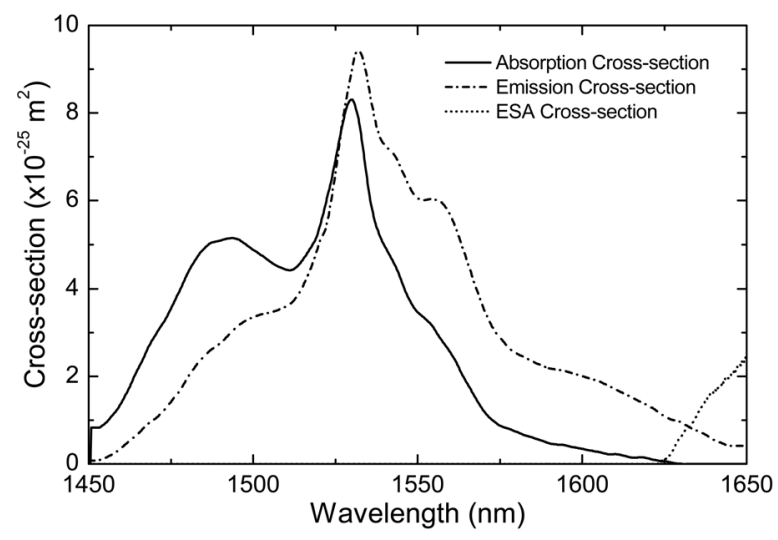

FIG. 2. The absorption, emission, and signal ESA cross-sections of the bismuth EDF used in this study [7, 14, 15].

TABLE 1. Parameters of the Bismuth oxide-based EDF used

\footnotetext{
${ }^{\mathrm{a}}$ Data from Ref. [16]

${ }^{\mathrm{b}}$ Data from Ref. [6]

${ }^{\mathrm{e}}$ Data from Ref. [5]

${ }^{\mathrm{f}}$ Data from Ref. [14] and Ref. [15]

${ }^{\mathrm{g}}$ Data from Ref. [14]
}

${ }^{\mathrm{c}, \mathrm{d}}$ Note that typical values for silica-based and tellurite-based $\mathrm{Er}^{3+}$-doped fibers from Ref. [10] and [17] were used since non-radiative lifetimes between the ${ }^{4} \mathrm{I}_{9 / 2}$ and ${ }^{4} \mathrm{I}_{11 / 2}$ states and between ${ }^{4} \mathrm{~S}_{3 / 2}$ and ${ }^{4} \mathrm{I}_{11 / 2}$ had not been reported for bismuth-based $\mathrm{Er}^{3+}$-doped fiber. 
peak emission and absorption cross-sections are $9.43 \times 10^{-25} \mathrm{~m}^{2}$ at $1532 \mathrm{~nm}$ and $8.31 \times 10^{-25} \mathrm{~m}^{2}$ at $1530 \mathrm{~nm}$, respectively. The signal ESA cross-section starts to appear at $1623 \mathrm{~nm}$ and increases monotonously up to $2.5 \times 10^{-25} \mathrm{~m}^{2}$ at 1650 $\mathrm{nm}$. The signal ESA cross-section $\sigma_{\text {signal }}^{E S A}$ was measured in our laboratory; further details on the signal ESA cross-section in bismuth oxide EDFs are fully described in [7]. The parameters of the bismuth oxide EDF used in this work are summarized in Table 1.

\section{ESTIMATION OF RELATIVE NUMBER OF PAIR IONS}

In order to estimate the relative number of paired ions $(k)$ we used a numerical prediction method based on the comparison between the experimentally measured gain and $\mathrm{NF}$ values and the numerically predicted ones. The experimental setup for the measurement of the signal gain and the NF for a $2 \mathrm{~m}$ long bismuth oxide EDF is shown in Fig. 3. The bismuth oxide EDF used in this investigation was a commercially available one from Asahi Glass company, model number T1L-201P/31024-01. The bismuth oxide EDF was pumped using a $160-\mathrm{mW}$ laser diode at $1480 \mathrm{~nm}$ using the forward pumping scheme. The tunable laser used as an input signal was a commercially available external cavity laser with a tuning range of $1490 \sim 1650$ $\mathrm{nm}$. The input signal power was fixed at $0 \mathrm{dBm}$. The signal gain and the NF were measured and numerically simulated at the signal wavelength of $1600 \mathrm{~nm}$ as the pump power was increased by $10-\mathrm{mW}$ steps.

Fig. 4(a) shows the numerically calculated gains for the four different $k$ values $(0,3,6.02$, and $9 \%)$, along with the experimentally measured ones. From the numerically calculated results, it is obvious that the signal gain significantly decreased as the $k$ value increased. After trying to find the best fitting theoretical gain curve for the experimentally measured unknown variable $k$ by using nonlinear least squares fitting, we found that the $k=6.02 \%$ led to the best fit. With respect to the NF, the same fitting procedure was used, as shown in Fig. 4(b). Interestingly, it was found from the numerically calculated NF curves that the NF remains fixed regardless of the relative number of paired ions. In addition, it was observed that the numerically calculated NF values agreed well with the experimentally

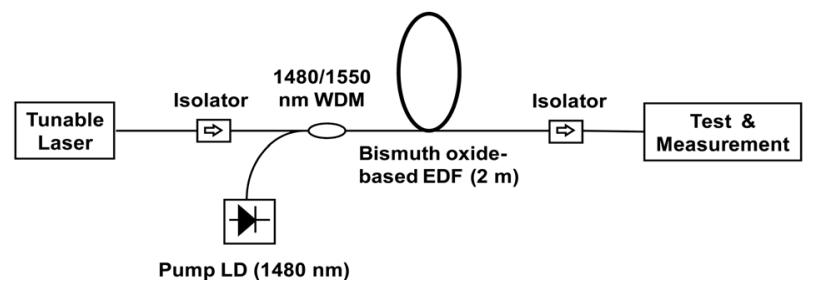

FIG. 3. An experimental schematic used for the estimation of the relative number of paired ions $(k)$. measured ones.

In order to confirm the estimated $k$ value $(6.02 \%)$ we carried out an output spectrum comparison. Fig. 5 shows the numerically calculated spectra for the various $k$ values along with the experimentally measured spectrum. It is

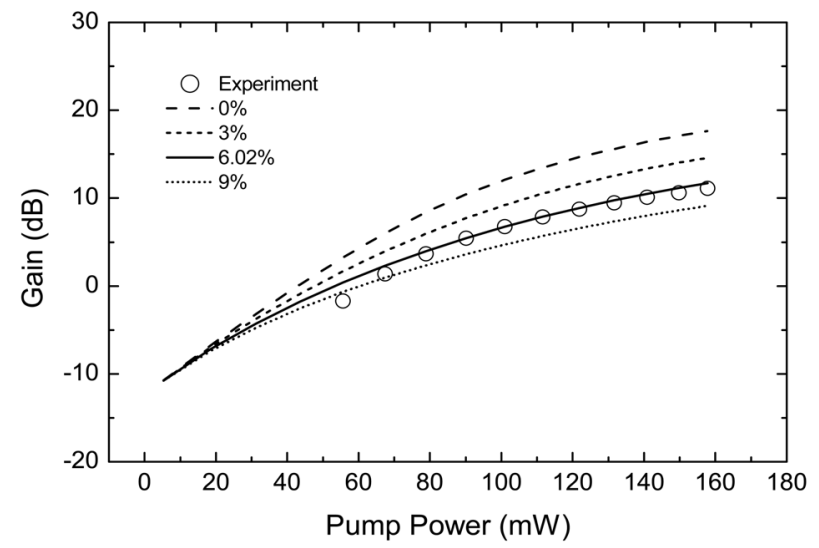

(a)

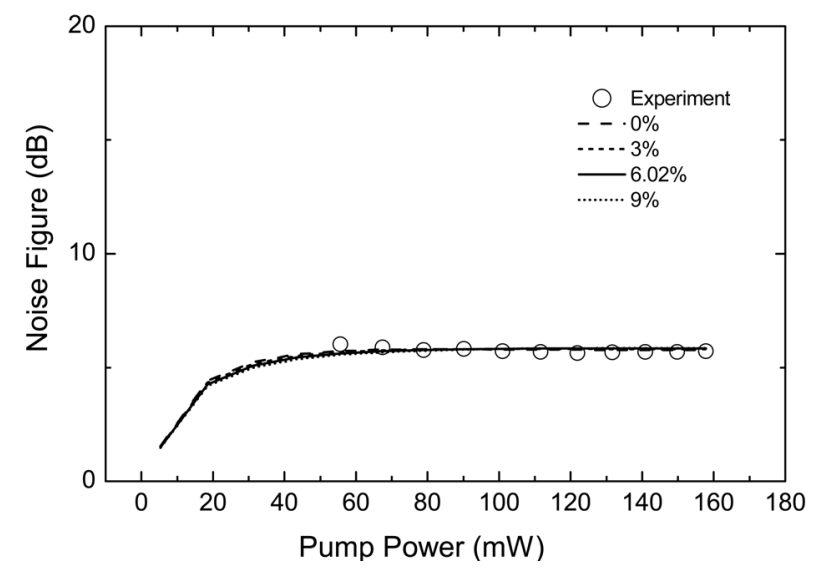

(b)

FIG. 4. The theoretically calculated gain (a) and NF (b) as a function of the pump power for various $k$ values (the relative number of paired ions); the experimentally measured gain (a) and NF (b) are also shown.

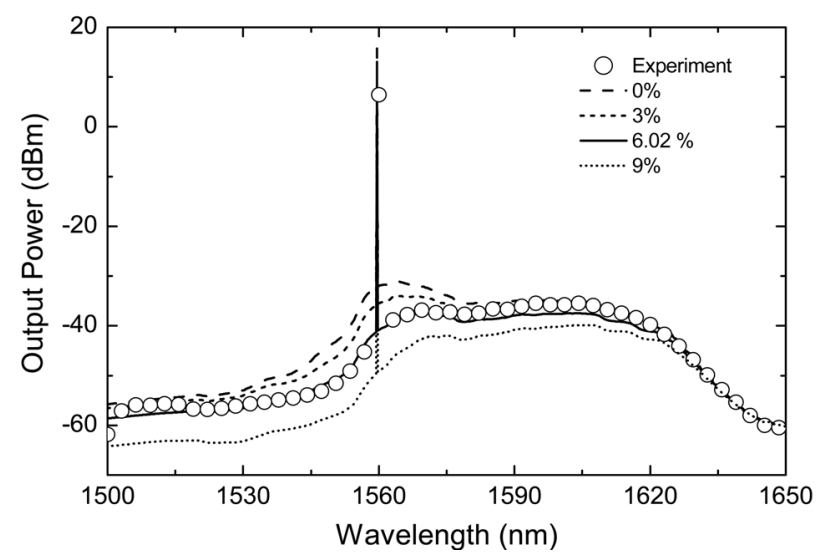

FIG. 5. The numerically calculated output spectra for various $k$ values together with the experimentally measured spectrum. 
clearly evident from the graph that $k=6.02 \%$ gives the best theoretical fit to the experimentally measured spectrum. This indicates that the relative number of paired ions $(k)$ in the bismuth oxide EDF used in this work is $\sim 6.02 \%$.

\section{IMPACTS OF PIQ ON GAIN AND NOISE FIGURE PERFORMANCE}

Finally, the impacts of the PIQ on the spectral gain and the NF were investigated. For this investigation, the experimental setup where the bismuth oxide-based EDF was pumped using a bidirectional pumping scheme is shown in Fig. 6. The forward pump power was $200 \mathrm{~mW}$ at $980 \mathrm{~nm}$; the backward pump power was $160 \mathrm{~mW}$ at $1480 \mathrm{~nm}$. The signal gain and the NF were measured and numerically simulated by sweeping the input signal beam wavelength from $1540 \mathrm{~nm}$ to $1635 \mathrm{~nm}$ by $5-\mathrm{nm}$ steps in order to analyze the impacts of the PIQ. The input signal power was fixed at $0 \mathrm{~dB} \mathrm{~m}$.

Fig. 7 shows the experimentally measured output spectrum along with the numerically calculated output spectrum based on $k=6.02 \%$. It was evident that our numerical calculation agreed well with the experiment, even in the complicated bidirectional pumping case. Fig. 8(a) shows the signal gains for three different cases: (i) the experi-

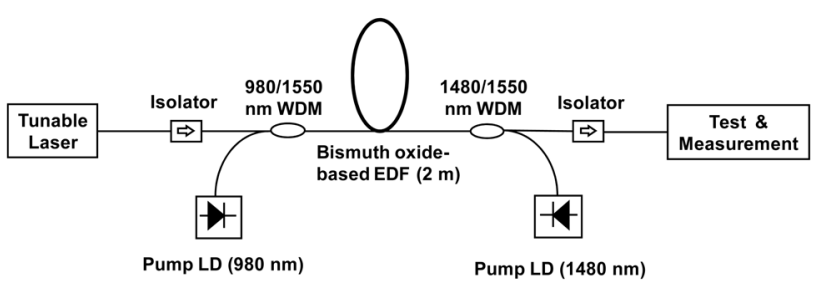

FIG. 6. An experimental schematic used for the investigation of the impacts of the PIQ on the gain and the NF.

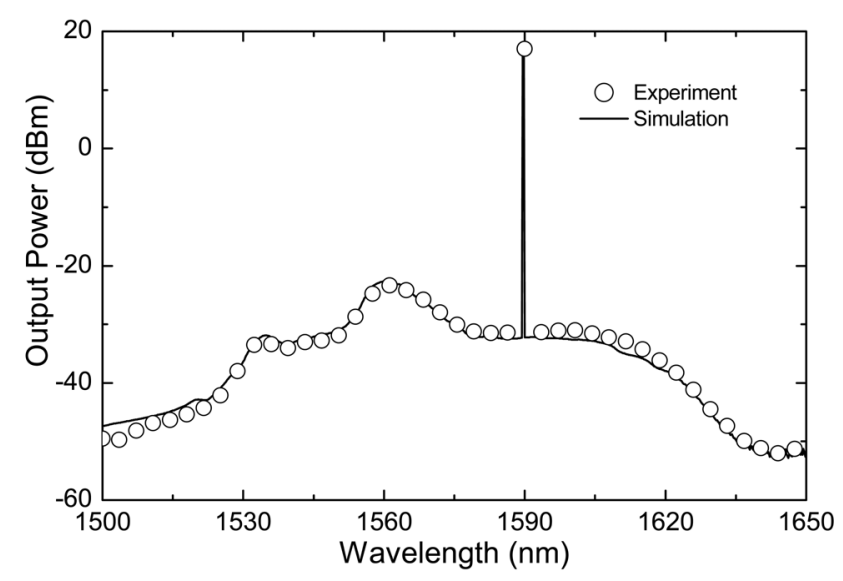

FIG. 7. The output spectrum comparison for two cases: (i) Experiment and (ii) Numerical simulation with the PIQ $k=$ $6.02 \%$.

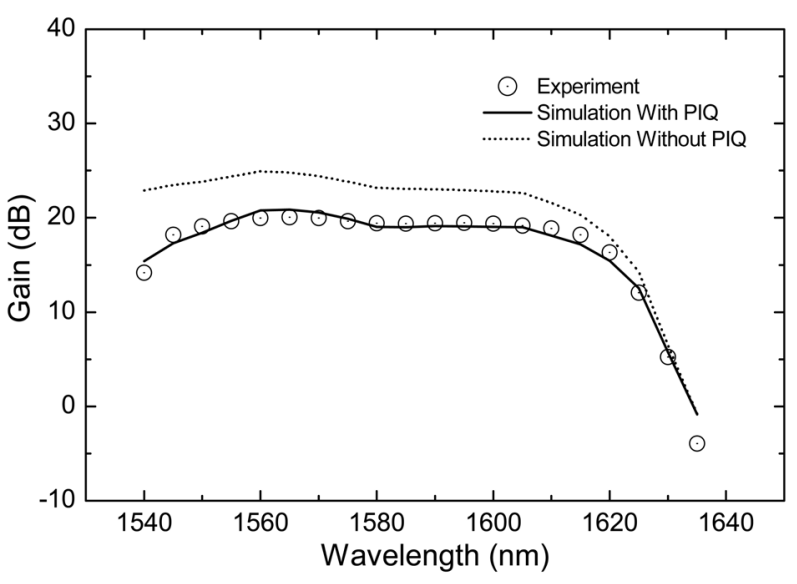

(a)

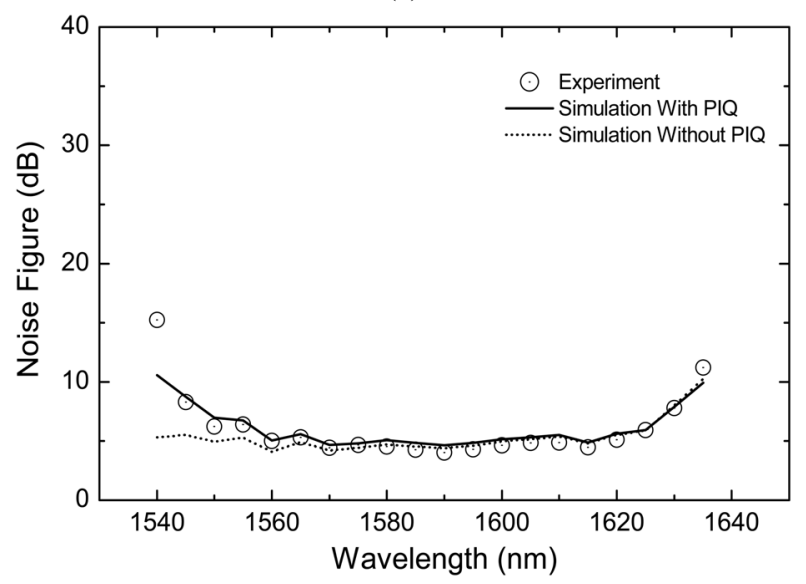

(b)

FIG. 8. The signal gain (a) and the NF (b) comparison for three cases: (i) the experiment, (ii) the numerical simulation with the PIQ $k=6.02 \%$, and (iii) the numerical simulation without the PIQ.

mental measurement, (ii) the numerical calculation with the PIQ, and (iii) the numerical calculation without the PIQ. The signal gain was observed to be reduced by $\sim 5 \mathrm{~dB}$ over a gain bandwidth of $\sim 90 \mathrm{~nm}$ from 1540 to $1630 \mathrm{~nm}$, compared to the value that is supposed to be achieved without the PIQ.

Unlike the gain degradation dependent on the PIQ, the NF exhibited a completely different behavior. The NF was observed to remain unchanged at the wavelengths from 1560 to 1630 irrespective of the relative number of paired ions. This result is consistent with the observation found in Section III, where the NF at $1600 \mathrm{~nm}$ under the forward pumping scheme was fixed regardless of the relative number of paired ions. However, non-negligible NF degradation caused by the PIQ was observed at the $1540 \sim 1560 \mathrm{~nm}$ wavelengths. Such a spectral dependence of the NF degradation caused by the PIQ is believed to be due to the relative increase of the ion population at the metastable state despite the population inversion being unchanged. This effect needs to be further investigated. 


\section{CONCLUSION}

We have theoretically and experimentally investigated the pair-induced quenching effect in a commercially available bismuth oxide-based EDFA. By using the comparison between the numerically calculated and the experimentally measured gain values, the relative number of paired ions is estimated to be $\sim 6.02 \%$ in the bismuth oxide EDF. The PIQ was observed to induce a $\sim 5-\mathrm{dB}$ gain reduction over a gain bandwidth of $\sim 90 \mathrm{~nm}$ from 1540 to $1630 \mathrm{~nm}$, compared to the value that is supposed to be achieved without the PIQ. Interestingly, NF degradation caused by the PIQ was observed only at the $1540 \sim 1560 \mathrm{~nm}$ wavelengths. This spectral dependence of the NF degradation caused by the PIQ needs to be further investigated.

\section{ACKNOWLEDGMENT}

This research work was supported by the Basic Science Research Program through the National Research Foundation of Korea (NRF) funded by the Ministry of Education, Science, and Technology (MEST), Republic of Korea (No.20100015230). This work was also supported by "Ultra-Precision Green Processing Technology Using Femtosecond Fiber Laser" Project funded by the Ministry of Knowledge Economy, Korea (No. 10037371).

\section{REFERENCES}

1. E. Desurvire, Erbium-doped Fiber Amplifiers: Principles and Applications (Wiley, New York, USA, 2002).

2. A. Mori, T. Sakamoto, K. Kobayashi, K. Shikano, K. Oikawa, K. Hoshino, T. Kanamori, Y. Ohishi, and M. Shimizu, "1.58- $\mu \mathrm{m}$ broad-band erbium-doped tellurite fiber amplifier," IEEE J. Lightwave Technol. 20, 794-799 (2002).

3. S. Ohara, N. Sugimoto, K. Ochiai, H. Hayashi, Y. Fukasawa, T. Hirose, T. Nagashima, and M. Reyes, "Ultra-wideband amplifiers based on $\mathrm{Bi}_{2} \mathrm{O}_{3}$-EDFAs," Opt. Fiber Technol. 10, 283-295 (2004).

4. A. J. G. Ellison, D. E. Goforth, B. N. Samson, J. D. Minelly, J. P. Trentelman, D. L. McEnroe, and B. P. Tyndell, "Extending the L-band to $1620 \mathrm{~nm}$ using MCS fiber," in Proc. Optical Fiber Communication Conference and Exhibit
(OFC2001) (Anaheim, USA, 2001), paper TuA2.

5. H. Hayashi, S. Ohara, N. Sugimoto, and S. Tanabe, "Effects of lanthanum and boron addition on suppression of cooperative upconversion in bismuth oxide-based erbium-doped fibers," Jpn. J. Appl. Phys. 46, 3452-3454 (2007).

6. H. Hayashi, S. Tanabe, and N. Sugimoto, "Quantitative analysis of optical power budget of bismuth oxide-based erbium-doped fiber," J. Lumin. 128, 333-340 (2008).

7. J. H. Shin and J. H. Lee, "Investigation of signal excited state absorption in bismuth-based erbium-doped fiber amplifier," J. Opt. Soc. Am. B. 27, 1452-1457 (2010).

8. P. Myslinski, D. Nguyen, and J. Chrostowski, "Effects of concentration on the performance of erbium-doped fiber amplifiers," IEEE J. Lightwave Technol. 15, 112-120 (1997).

9. J. L. Wagener, P. F. Wysocki, M. J. F. Digonnet, and H. J. Shaw, "Modeling of ion pairs in erbium-doped fiber amplifiers," Opt. Lett. 19, 347-349 (1994).

10. C. R. Giles and E. Desurvire, "Propagation of signal and noise in concatenated erbium-doped fiber optical amplifiers," IEEE J. Lightwave Technol. 9, 147-154 (1991).

11. Y. O. Barmenkov, A. V. Kir’yanov, A. D. Guzmán-Chávez, J.-L. Cruz, and M. V. Andrés, "Excited-state absorption in erbium-doped silica fiber with simultaneous excitation at 977 and 1531 nm,” J. Appl. Phys. 106, 083108 (2009).

12. A. P. López-Barbero, W. A. Arellano-Espinoza, H. L. Fragnito, and H. E. Hernández-Figueroa, "Tellurite-based optical fiber amplifier analysis using the finite-element method," Microw. Opt. Technol. Lett. 25, 103-107 (2000).

13. C. Jiang, W. Hu, and Q. Zeng, "Numerical analysis of concentration quenching model of $\mathrm{Er}^{3+}$-doped phosphate fiber amplifier," IEEE J. Quantum Electron. 39, 1266-1271 (2003).

14. Asahi Glass Company Technical Bulletin, http://www.agc.co.jp/ english/biedf/bi5web.pdf.

15. S. Tanabe, N. Sugimoto, S. Ito, and T. Hanada, "Broad-band $1.5 \mu \mathrm{m}$ emission of $\mathrm{Er}^{3+}$ ions in bismuth-based oxide glasses for potential WDM amplifier," J. Lumin. 87-89, 670-672 (2000).

16. H. Hayashi, N. Sugimoto, and S. Tanabe, "High-performance and wideband amplifier using bismuth-oxide-based EDF with cascade configurations," Opt. Fiber Technol. 12, 282-287 (2006).

17. Y. Hu, S. Jiang, G. Sorbello, T. Luo, Y. Ding, B. C. Hwang, J. H. Kim, H. J. Seo, and N. Peyghambarian, "Numerical analyses of the population dynamics and determination of the upconversion coefficients in a new high erbium-doped tellurite glass," J. Opt. Soc. Am. B. 18, 1928-1934 (2001). 